\title{
Evaluation and Development of Environmental and Health Efficiency: Case of V4 Countries
}

\author{
Roman LACKO* - Peter MARKOVIČ** _ Slávka ŠAGÁTOVÁ** _ \\ Zuzana HAJDUOVÁ**
}

\begin{abstract}
The study deals with the topic of evaluation and comparison of environmental efficiency and efficiency of health care systems in the V4 countries during the period from 2009 to 2017. The uniqueness of this study lies in the comparison of two different types of efficiencies and in the selection of the object of research. The aim of this paper was to examine the possible relationship between environmental and health efficiency in the V4 countries and to reveal the common determinants of these efficiencies. We implemented the method of two-step Data envelopment analysis, in which, we determined the values of input CCR and $B C C$ health and environmental efficiency in the first step and then in the second step, we used the method of truncated regression to verify the effects of selected determinants of computed efficiencies in V4 countries. Based on these analyses, we found that although there is no significant relationship between the values of health efficiency and environmental efficiency, some determinants significantly affect both types of efficiencies.
\end{abstract}

Keywords: healthcare, environment, efficiency, DEA, truncated regression JEL Classification: C67, I15, I18, Q50

DOI: https://doi.org/10.31577/ekoncas.2020.10.04

\footnotetext{
* Roman LACKO, University of Economics in Bratislava, Faculty of Business Economics with seat in Košice, Department of Economy, Tajovského 13, 04130 Košice, Slovakia; e-mail: roman.lacko@euke.sk

** Peter MARKOVIČ - Slávka ŠAGÁTOVÁ - Zuzana HAJDUOVÁ, University of Economics in Bratislava, Faculty of Business Managment, Department of Business Finance, Dolnozemská cesta 1,85235 Bratislava, Slovakia; e-mail: peter.markovic@euba.sk; slavka.sagatova@euba.sk; zuzana.hajduova@euba.sk
} 


\section{Introduction}

The environment and health care have much in common. One of the most important entities that it directly affects is the person. Health and the environment are among the greatest research challenges of this time.

Environmental efficiency has recently been a much-discussed area, especially at the country level (Madaleno, Moutinho and Robaina, 2016; Moutinho, Madaleno and Robaina, 2017). Emissions of $\mathrm{CO}_{2}$ are the main source of global warming and its negative effects (Sueyoshi and Goto, 2010). Industry and manufacturing are producing microparticles which directly harms people's health, such as $\mathrm{PM}_{2.5}$ and $\mathrm{PM}_{10}$ (Ji, Yao and Long, 2018; Liu et al., 2018). The efficiencies between countries differs significantly (Woo et al., 2015; Zhou, Ang and Poh, 2008). There are also technological differences among countries in political grouping such as the European Union (Kounetas, 2015) and in regions of individual countries ((Yang et al., 2015). Firstly, the efficiency of environment needs to be computed and then the researchers should propose policy changes. For the purposes of environmental efficiency or performance measurement, the economic variables seem to be the most important (Arbolino et al., 2018). Some studies have shown that higher efficient countries are improving their efficiency more than middle or low-income countries and economic development has positive impact on environmental efficiency ( $\mathrm{Li}$ and Wang, 2014). So, the policies must be considered according to economic performance in smaller groupings. In last years, there is a high pressure on countries to improve the state of the environment. Thus, a lot of eco-innovations are implemented and it seems, they have also positive economic impact (Cai and $\mathrm{Li}, 2018$ ). There are also other determinants of environmental efficiency such as energy consumption and energy efficiency, or bioenergy efficiency (Alsaleh, Abdul-Rahim and Mohd-Shahwahid, 2017). Improving energy efficiency could lead to improvement of environmental efficiency (Tajudeen, Wossink and Banerjee, 2018). In this study, we are assessing the environmental efficiency of Visegrad 4 (V4) countries. As they do not belong to the "original" EU-15 countries, and some studies (Duman and Kasman, 2018) propose that new members have smaller potential to reduce emissions, but could simultaneously reduce energy use and improve GDP, we have to focus some policies on smaller groupings. Such the V4 countries are. The state of the environment directly affects people's health, so it does affect the health care of individual countries. The higher number of ill people means the higher costs in health care. One can state that there could be relationship between environmental and health care efficiency.

Large number of studies are examining the efficiency of health care providers, such as hospitals (Chuang, Chang and Lin, 2011; Nayar and Ozcan, 2008; O'Neill et al., 2008), but also among countries of the world (Afonso and St. Aubyn, 2011; 
Hadad, Hadad and Simon-Tuval, 2013; Hsu, 2013; Kaya Samut and Cafri, 2016) and between regions (Halkos and Tzeremes, 2011). However, the number of studies, which are assessing the efficiency internationally, is relatively low to the number of studies assessing individual countries or hospitals. There are differences in health care efficiency among countries as it is in the case of environmental efficiency (Hadad, Hadad and Simon-Tuval, 2013). Any study has brought the concise and clear comparison between environmental and health care efficiencies to this time.

As there are just a few studies on each type of efficiency at the level of the V4 group, we decided to not only measure and evaluate the health care and environmental efficiency. The goal is to compare and verify the possible relationship between environmental and health care efficiency between Poland, Slovakia, the Czech Republic and Hungary, which are linked to historical events. We will also propose models for evaluating the determinants of both types of efficiency. Most of the above-mentioned studies use as the main method the Data envelopment analysis. This method is mostly used in the areas of environmental and health efficiency (Emrouznejad and Yang, 2018; Hollingsworth, 2003; Liu et al., 2013; Mardani et al., 2017).

\section{Methods, Methodology and Research Data}

In this section, we provide an overview of the procedures, methods, and variables used to perform the analysis. Based on a review of the literature, we have identified that the most used method for evaluating efficiency in health care but also in the environment is Data envelopment analysis (DEA). Its theoretical foundations were laid by (Farrell, 1957) and subsequently developed in many other studies. (Cooper, Seiford and Tone, 2007) aided significant theoretical development. They proposed models that assume either constant returns to scale, the so-called CCR DEA models (1) and models that assume variable returns to scale, so-called BCC DEA models (2). In this paper, we will use exclusively input-oriented DEA models, as in terms of the models used, the influence of inputs and not outputs can be assumed. Moreover, in terms of efficiency results, this is irrelevant, as output efficiency is only the inverse of input efficiency. The difference is only in the slacks (Charnes et al., 1984; Charnes et al., 2013; Cooper, Seiford and Tone, 2007).

$$
\begin{gathered}
\min _{\theta_{B}, \lambda} \theta_{B} \\
\text { s.t. } \theta_{B} x_{o}-X \lambda \geq 0 \\
Y \lambda \geq y_{o} \\
\lambda \geq 0
\end{gathered}
$$




$$
\begin{gathered}
\min _{\theta_{B}, \lambda} \theta_{B} \\
\text { s.t. } \theta_{B} x_{o}-X \lambda \geq 0 \\
Y \lambda \geq y_{o} \\
e \lambda=1 \\
\lambda \geq 0
\end{gathered}
$$

where $\theta_{B}$ is a real efficiency value, $X=\left(x_{j}\right) \in R^{m \times n}, Y=\left(y_{j}\right) \in R^{s \times n}$ are a given set of data, $e$ is row vector, in which, all elements are equal to $1, \lambda \in R^{n}$ - non-negative vector, and $x_{o}$ and $y_{o}$ are positive input and output vectors.

Input efficiency values are in the range $\langle 0 ; 1\rangle$ and the subject who achieved a value of 1 is efficient. In this study, we will use the two-step DEA method, which will help us meet the aim of the study. This method is often used in other studies and its results show good predictive power (Afonso and St. Aubyn, 2011; Lacko and Hajduová, 2018; Liu et al., 2013; Tajudeen, Wossink and Banerjee, 2018). In the first step, we calculate the CCR and BCC input efficiencies of the environment and health care. These values we use in the second step as a dependent variable to evaluate the impact of selected exogenous variables. The DEA method is a deterministic method, so it brings with it several shortcomings, including failure to consider statistical bias. Based on this fact, it can be concluded that performing any regression analysis with a deterministically calculated efficiency value would be incorrect. Therefore, in this study we will use the approach proposed by Simar and Wilson (2007), who proposed a resampling of efficiency values according to a specific algorithm. Such an adjustment requires the use of the double bootstrap method. Subsequently, the method of truncated regression as proposed by (Simar and Wilson, 2007) is used. Some authors (Kaya Samut and Cafri, 2016; Li and Wang, 2014; McDonald, 2009; Wang, Han and Yin, 2016) also use Tobit regression, but in some articles, it does not show sufficient explanatory power (Lacko and Hajduová, 2018).

The object of research in this study is the political grouping of the Visegrad Four (V4) countries, which is located in the middle of Europe and consists of four countries that have similar political and economic development. These are the countries of the former Soviet Union. The countries that belong to the V4 group are Hungary, Poland, the Czech Republic and Slovakia. There are studies, which compares V4 countries in the field of healthcare or environment, e.g. Grausová, Hužvár and Štrangfeldová, (2014) measured healthcare efficiency of V4 countries. There are also studies which compares countries on macro economical basis such as Koišová et al. (2019) Comparison of V4 countries is from the point of view of the goal of this study unique, as the studies that have carried 
out similar analysis have focused on the European Union (EU) as the whole, rather than on its micro political groupings. However, comparing weaker countries with economic leaders such as Germany or France may not provide a sufficient understanding of local differences in Europe's regions. We examine the efficiency during the period from 2009 to 2017. There were no newer data for some variables. We obtained data for our analysis from The World Bank (2020) and Eurostat (2020) databases. This is how we obtained the panel of 36 Decision-making units (DMU) and since we will use the Window approach (Halkos and Tzeremes, 2009; Wang, Yu and Zhang, 2013), each country in each year will be considered as an individual DMU.

Based on a literature review, we selected the input and output variables of the DEA models for both environmental efficiency and health efficiency. Table 1 lists the input and output variables for both types of efficiency. Table 1 also shows the units of presented variables and basic statistical indicators.

As inputs for the calculation of environmental efficiency, we used the most frequently used variables (Alsaleh, Abdul-Rahim and Mohd-Shahwahid, 2017; Halkos and Petrou, 2019; Madaleno, Moutinho and Robaina, 2016; Moutinho, (Madaleno, Moutinho and Robaina, 2016; Moutinho, Madaleno and Robaina, 2017), which express the basic economic resources, namely land, labour and capital. For environmental efficiency, we use one desirable output - GDP per capita and one undesirable output $-\mathrm{CO}_{2}$ emissions per capita, which are also often used in previous studies. We will treat the undesirable output as negative input (Bian and Yang, 2010; Shi, Bi and Wang, 2010).

To calculate the efficiency of health care, we used indicators of the capacity of health systems of the studied countries as input variables - Hospital beds and Health personnel and an indicator of economic resources - Health expenditures. As output variables, we chose indicators related to the health status of the population - Health life years and Life expectancy at birth and the indicator of health capacity utilization - Hospital discharges. These indicators are among the most used in assessing the efficiency of health care in countries and groupings (Hadad, Hadad and Simon-Tuval, 2013).

Subsequently, we created two models for evaluating the determinants of environmental efficiency and health efficiency. In Table 2, we can find indicators related to economic indicators - Energy consumption, Unemployment; environmental pollution - $\mathrm{PM}_{2.5}$ air pollution, Waste generated, Recycling - composting and digestion; agriculture - Area under organic farming, Sales of fertilizers; transport - Freight transport and Air transport. All these variables can affect positively but also negatively both the health of the population and the health of the environment. 
T a ble 1

Description of Input and Output Variables of DEA Models

\begin{tabular}{|c|c|c|c|c|c|c|c|}
\hline \multirow{2}{*}{\multicolumn{2}{|c|}{ First step DEA }} & \multicolumn{3}{|c|}{ Inputs } & \multicolumn{3}{|c|}{ Outputs } \\
\hline & & \multicolumn{6}{|c|}{ Environmental efficiency } \\
\hline & Variable & $\begin{array}{l}\text { Agrable } \\
\text { land }\end{array}$ & $\begin{array}{l}\text { Gross capital } \\
\text { formation }\end{array}$ & Labor force & $\begin{array}{c}\mathrm{CO}_{2} \\
\text { emissions } \\
\text { (undesirable) }\end{array}$ & GDP & \\
\hline Country & Unit & square km & USD & Total & $\begin{array}{c}\text { tones per } \\
\text { capita }\end{array}$ & $\begin{array}{c}\text { USD per } \\
\text { capita }\end{array}$ & \\
\hline $\begin{array}{l}\text { Czech } \\
\text { Republic }\end{array}$ & $\begin{array}{l}\text { Mean } \\
\text { StDev } \\
\text { Min } \\
\text { Max } \\
\end{array}$ & $\begin{array}{r}77228.89 \\
13.70 \\
77210.00 \\
77250.00 \\
\end{array}$ & $\begin{array}{r}26.35 \\
0.88 \\
24.67 \\
27.96 \\
\end{array}$ & $\begin{array}{r}5309597.67 \\
62411.68 \\
5232722.00 \\
5418917.00 \\
\end{array}$ & $\begin{array}{r}12.73 \\
0.49 \\
12.20 \\
13.50 \\
\end{array}$ & $\begin{array}{r}19690.72 \\
1057.33 \\
17715.62 \\
21717.46 \\
\end{array}$ & \\
\hline Hungary & \begin{tabular}{|l|} 
Mean \\
StDev \\
Min \\
Max
\end{tabular} & $\begin{array}{r}90530.00 \\
0.00 \\
90530.00 \\
90530.00\end{array}$ & $\begin{array}{r}21.38 \\
1.39 \\
19.33 \\
27.96\end{array}$ & $\begin{array}{r}4465235.89 \\
164187.35 \\
4258571.00 \\
5418917.00\end{array}$ & $\begin{array}{r}6.27 \\
0.27 \\
5.80 \\
12.40\end{array}$ & \begin{tabular}{r|}
13473.79 \\
632.22 \\
12651.57 \\
20379.90
\end{tabular} & \\
\hline Poland & \begin{tabular}{|l|} 
Mean \\
StDev \\
Min \\
Max \\
\end{tabular} & $\begin{array}{r}306220.00 \\
34.96 \\
306190.00 \\
306280.00\end{array}$ & $\begin{array}{r}20.50 \\
0.96 \\
18.98 \\
22.44\end{array}$ & $\begin{array}{r}18233721.78 \\
194044.28 \\
17832669.00 \\
18466308.00\end{array}$ & $\begin{array}{r}10.59 \\
0.26 \\
10.20 \\
11.00\end{array}$ & $\begin{array}{r}13128.45 \\
860.67 \\
11527.59 \\
14347.91 \\
\end{array}$ & \\
\hline $\begin{array}{l}\text { Slovak } \\
\text { Republic }\end{array}$ & \begin{tabular}{|l|} 
Mean \\
StDev \\
Min \\
Max \\
\end{tabular} & $\begin{array}{r}48085.11 \\
4.23 \\
48080.00 \\
48090.00 \\
\end{array}$ & $\begin{array}{r}22.59 \\
1.61 \\
20.50 \\
25.24 \\
\end{array}$ & $\begin{array}{r}2717780.56 \\
31386.79 \\
2672217.00 \\
2763640.00 \\
\end{array}$ & $\begin{array}{l}8.06 \\
0.37 \\
7.50 \\
8.60 \\
\end{array}$ & $\begin{array}{r}17365.60 \\
843.10 \\
16309.07 \\
18670.93 \\
\end{array}$ & \\
\hline & & \multicolumn{6}{|c|}{ Healthcare efficiency } \\
\hline & Variable & $\begin{array}{l}\text { Hospital } \\
\text { beds }\end{array}$ & $\begin{array}{c}\text { Health } \\
\text { personnel }\end{array}$ & $\begin{array}{l}\text { Healthcare } \\
\text { expenditures }\end{array}$ & $\begin{array}{c}\text { Healthy life } \\
\text { years }\end{array}$ & $\begin{array}{c}\text { Life } \\
\text { expectancy } \\
\text { at birth }\end{array}$ & $\begin{array}{c}\text { Hospital } \\
\text { discharges }\end{array}$ \\
\hline Country & Unit & $\begin{array}{c}\text { per } 100000 \\
\text { inhabitants }\end{array}$ & $\begin{array}{l}\text { per } 100000 \\
\text { inhabitants }\end{array}$ & $\%$ of GDP & years & years & $\begin{array}{c}\text { per } \\
100000 \\
\text { inhabitants } \\
\end{array}$ \\
\hline $\begin{array}{l}\text { Czech } \\
\text { Republic }\end{array}$ & $\begin{array}{l}\text { Mean } \\
\text { StDev } \\
\text { Min } \\
\text { Max }\end{array}$ & $\begin{array}{r}686.32 \\
23.67 \\
662.74 \\
729.52\end{array}$ & $\begin{array}{r}195.57 \\
7.23 \\
186.52 \\
211.17\end{array}$ & $\begin{array}{l}7.26 \\
0.28 \\
6.93 \\
7.81\end{array}$ & $\begin{array}{r}63.80 \\
0.78 \\
62.40 \\
65.00\end{array}$ & $\begin{array}{r}78.37 \\
0.58 \\
77.40 \\
79.10\end{array}$ & $\begin{array}{r}20322.56 \\
325.89 \\
19814.00 \\
20900.00\end{array}$ \\
\hline Hungary & \begin{tabular}{|l|} 
Mean \\
StDev \\
Min \\
Max \\
\end{tabular} & $\begin{array}{r}706.21 \\
8.14 \\
698.43 \\
719.65 \\
\end{array}$ & $\begin{array}{r}164.61 \\
14.87 \\
128.96 \\
211.17 \\
\end{array}$ & $\begin{array}{l}7.24 \\
0.22 \\
6.88 \\
7.81 \\
\end{array}$ & $\begin{array}{r}59.80 \\
0.86 \\
58.20 \\
65.00 \\
\end{array}$ & $\begin{array}{r}75.47 \\
0.59 \\
74.40 \\
79.10 \\
\end{array}$ & $\begin{array}{r}20280.33 \\
477.53 \\
19450.00 \\
21237.00 \\
\end{array}$ \\
\hline Poland & \begin{tabular}{|l|} 
Mean \\
StDev \\
Min \\
Max \\
\end{tabular} & $\begin{array}{r}662.97 \\
1.60 \\
660.84 \\
666.54 \\
\end{array}$ & $\begin{array}{r}200.12 \\
33.54 \\
149.10 \\
246.82 \\
\end{array}$ & $\begin{array}{l}6.38 \\
0.13 \\
6.20 \\
6.59 \\
\end{array}$ & $\begin{array}{r}63.06 \\
0.65 \\
62.30 \\
64.60 \\
\end{array}$ & $\begin{array}{r}77.13 \\
0.67 \\
75.90 \\
78.00\end{array}$ & $\begin{array}{r}16893.56 \\
684.81 \\
16172.00 \\
18152.00 \\
\end{array}$ \\
\hline $\begin{array}{l}\text { Slovak } \\
\text { Republic }\end{array}$ & $\begin{array}{l}\text { Mean } \\
\text { StDev } \\
\text { Min } \\
\text { Max } \\
\end{array}$ & $\begin{array}{r}599.00 \\
28.83 \\
574.73 \\
654.39 \\
\end{array}$ & $\begin{array}{r}160.80 \\
5.62 \\
152.58 \\
167.47 \\
\end{array}$ & $\begin{array}{l}7.31 \\
0.43 \\
6.74 \\
7.96 \\
\end{array}$ & $\begin{array}{r}54.07 \\
1.59 \\
52.00 \\
57.00 \\
\end{array}$ & $\begin{array}{r}76.47 \\
0.67 \\
75.30 \\
77.30 \\
\end{array}$ & $\begin{array}{r}19163.67 \\
417.88 \\
18368.00 \\
19733.00 \\
\end{array}$ \\
\hline
\end{tabular}

Source: Own processing according to data collected from Eurostat (2020) and World Bank (2020).

Models (3) and (4) that we have designed are stated as follows:

$\widehat{\widehat{\delta_{E N V}}}=\beta_{0}+\beta_{1}$ EnergyCons $+\beta_{2}$ PM $2.5+\beta_{3}$ Unempl.$+\beta_{4}$ Waste $+\beta_{5}$ Recycling + $+\beta_{6}$ AreaOrgFarm $+\beta_{7}$ SalesFert $+\beta_{8}$ FreightTransp $+\beta_{9}$ AirTransp $+\varepsilon_{i}$ 
where $\widehat{\widehat{\delta_{E N V}}}$ is the double bootstrap BCC value of the environmental efficiency, which is calculated according to the second algorithm proposed by Simar and Wilson (2007).

$$
\begin{aligned}
& \widehat{\widehat{\delta_{\text {HEALTH }}}}=\beta_{0}+\beta_{1} \text { EnergyCons }+\beta_{2} P M_{2.5}+\beta_{3} \text { Unempl } .+\beta_{4} \text { Waste }+ \\
& +\beta_{5} \text { Recycling }+\beta_{6} \text { AreaOrgFarm }+\beta_{7} \text { SalesFert }+\beta_{8} \text { FreightTransp }+ \\
& +\beta_{9} \text { AirTransp }+\varepsilon_{i}
\end{aligned}
$$

where $\overline{\widehat{\delta_{\text {HEALTH }}}}$ is the double bootstrap BCC value of the health care efficiency, which is calculated according to the second algorithm proposed by Simar and Wilson (2007).

In the study, we use only BCC efficiencies as dependent variables due to the assumption of variable returns to scale of technologies in selected countries. Table 2 lists the units of variables and selected statistical indicators.

\begin{tabular}{|c|c|c|c|c|c|c|c|c|c|c|}
\hline \multirow{2}{*}{$\stackrel{\Xi}{\Xi}$} & Var. & 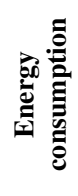 & 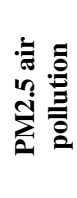 & 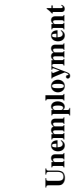 & 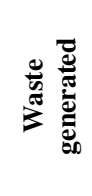 & 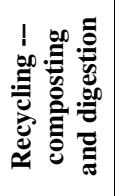 & 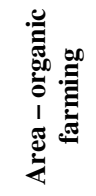 & 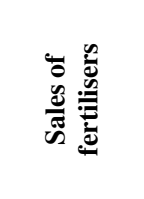 & 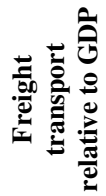 & 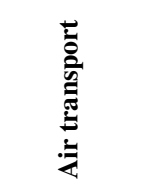 \\
\hline & Unit & 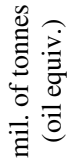 & 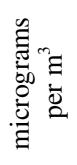 & $2^{2}$ &  & 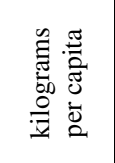 & 世葛 & 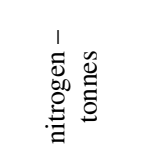 & 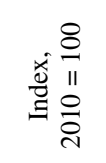 & 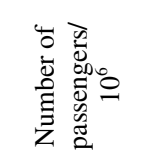 \\
\hline \multirow{4}{*}{ 专 } & Mean & 24.60 & 18.35 & 5.84 & 319.89 & 11.78 & 13.12 & 313900.22 & 106.31 & 12840482.44 \\
\hline & StDev & 0.56 & 1.70 & 1.45 & 12.38 & 6.86 & 1.01 & 36580.86 & 7.20 & 1317272.34 \\
\hline & Min & 23.58 & 16.07 & 2.89 & 307.00 & 5.00 & 10.60 & 246000.00 & 93.80 & 11742352.00 \\
\hline & Max & 25.50 & 20.68 & 7.28 & 344.00 & 25.00 & 14.09 & 353733.00 & 116.50 & 16245554.00 \\
\hline \multirow{4}{*}{ 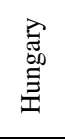 } & Mean & 17.23 & 17.53 & 8.58 & 391.22 & 20.89 & 2.66 & 316613.00 & 98.28 & 9589463.44 \\
\hline & StDev & 3.69 & 1.17 & 3.10 & 43.38 & 5.70 & 5.64 & 32496.24 & 7.69 & 2725501.92 \\
\hline & Min & 16.47 & 16.07 & 2.89 & 307.00 & 9.00 & 2.30 & 259000.00 & 95.30 & 8081067.00 \\
\hline & Max & 25.50 & 19.36 & 11.17 & 430.00 & 25.00 & 14.09 & 353733.00 & 116.50 & 16245554.00 \\
\hline \multirow{4}{*}{$\begin{array}{l}\vec{E} \\
\frac{\vec{J}}{0} \\
\text { 20 }\end{array}$} & Mean & 64.62 & 23.85 & 8.38 & 305.00 & 11.22 & 3.84 & 1087938.22 & 102.97 & 25079369.44 \\
\hline & StDev & 2.85 & 2.37 & 1.77 & 15.63 & 7.02 & 0.71 & 38722.63 & 4.29 & 6400735.25 \\
\hline & Min & 61.52 & 20.88 & 4.89 & 272.00 & 4.00 & 2.30 & 1003850.00 & 94.80 & 17046899.00 \\
\hline & Max & 70.97 & 27.18 & 10.33 & 319.00 & 22.00 & 4.65 & 1150668.00 & 110.60 & 37684668.00 \\
\hline \multirow{4}{*}{ 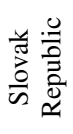 } & Mean & 10.60 & 19.58 & 12.30 & 324.67 & 18.22 & 8.93 & 120298.44 & 97.73 & 1881621.78 \\
\hline & StDev & 0.47 & 1.67 & 2.06 & 22.94 & 7.22 & 0.75 & 15211.87 & 1.99 & 260898.48 \\
\hline & Min & 9.96 & 17.35 & 8.13 & 304.00 & 11.00 & 7.50 & 82000.00 & 94.30 & 1557149.00 \\
\hline & Max & 11.54 & 21.77 & 14.38 & 378.00 & 33.00 & 9.90 & 135553.00 & 101.20 & 2402651.00 \\
\hline
\end{tabular}

T a b le 2

\section{Description of Explanatory Variables}

Source: Own processing according to data collected from Eurostat (2020) and World Bank (2020). 
Moreover we will test the hypothesis, whether there is any correlation between health care and environmental efficiency in the selected group of countries. For this purpose, we will use the Spearman correlation coefficient.

\section{Results}

In this section, we will focus on the results of modelling using the DEA twostep method. Computations presented in this section were computed using programming software RStudio. In the first step, it was necessary to calculate the values of DEA CCR and BCC efficiencies for individual countries for the observed period. Table 3 shows the results obtained using the DEA CCR model.

T a b le 3

\section{Results of DEA CCR Model}

\begin{tabular}{|l|c|c|c|c|c|c|c|c|c|}
\hline Country & $\mathbf{2 0 0 9}$ & $\mathbf{2 0 1 0}$ & $\mathbf{2 0 1 1}$ & $\mathbf{2 0 1 2}$ & $\mathbf{2 0 1 3}$ & $\mathbf{2 0 1 4}$ & $\mathbf{2 0 1 5}$ & $\mathbf{2 0 1 6}$ & $\mathbf{2 0 1 7}$ \\
\hline CR_EE & 1.0000 & 1.0000 & 1.0000 & 0.9315 & 1.0000 & 0.9265 & 0.7548 & 0.8662 & 0.9590 \\
CR_HE & 0.9745 & 1.0000 & 0.9915 & 0.9959 & 0.9762 & 0.9923 & 0.9934 & 0.9985 & 0.9709 \\
HU_EE & 0.7387 & 0.7303 & 0.7965 & 0.7666 & 0.7505 & 0.6974 & 0.6230 & 0.6993 & 0.7260 \\
HU_HE & 1.0000 & 0.9453 & 0.9366 & 0.9621 & 0.9532 & 0.9774 & 0.9739 & 0.9592 & 0.9745 \\
PL_EE & 0.6931 & 0.7439 & 0.7566 & 0.7789 & 1.0000 & 0.8720 & 0.7621 & 0.8449 & 0.9559 \\
PL_HE & 1.0000 & 1.0000 & 1.0000 & 1.0000 & 0.9909 & 1.0000 & 0.9961 & 1.0000 & 0.9971 \\
SR_EE & 1.0000 & 1.0000 & 1.0000 & 0.9851 & 1.0000 & 1.0000 & 0.8766 & 0.8888 & 0.9496 \\
SR_HE & 0.9608 & 0.9569 & 1.0000 & 1.0000 & 1.0000 & 1.0000 & 1.0000 & 1.0000 & 1.0000 \\
\hline
\end{tabular}

Note: CR - Czech Republic, HU - Hungary, PL - Poland, SR - Slovak Republic, EE - Environmental efficiency, HE - Healthcare efficiency.

Source: Own processing according to data collected from Eurostat (2020) and World Bank (2020).

In terms of environmental efficiency, Hungary and Poland are the least efficient countries during the period under review. The only difference, however, is that efficiency in Hungary has stagnated and in Poland it has begun to increase in recent years.

Table 4

\section{Results of DEA BCC Model}

\begin{tabular}{|l|c|c|c|c|c|c|c|c|c|}
\hline Country & $\mathbf{2 0 0 9}$ & $\mathbf{2 0 1 0}$ & $\mathbf{2 0 1 1}$ & $\mathbf{2 0 1 2}$ & $\mathbf{2 0 1 3}$ & $\mathbf{2 0 1 4}$ & $\mathbf{2 0 1 5}$ & $\mathbf{2 0 1 6}$ & $\mathbf{2 0 1 7}$ \\
\hline CR_EE & 1.0000 & 1.0000 & 1.0000 & 0.9565 & 1.0000 & 0.9278 & 0.7778 & 0.8950 & 0.9628 \\
CR_HE & 1.0000 & 1.0000 & 0.9932 & 0.9966 & 0.9943 & 1.0000 & 1.0000 & 1.0000 & 1.0000 \\
HU_EE & 0.9632 & 0.9482 & 0.9658 & 1.0000 & 0.9348 & 0.8425 & 0.8462 & 0.9167 & 0.8658 \\
HU_HE & 1.0000 & 0.9454 & 0.9385 & 0.9734 & 0.9544 & 0.9780 & 0.9826 & 0.9609 & 0.9816 \\
PL_EE & 0.9248 & 0.8932 & 0.8527 & 0.9065 & 1.0000 & 0.9445 & 0.9294 & 0.9699 & 0.9681 \\
PL_HE & 1.0000 & 1.0000 & 1.0000 & 1.0000 & 0.9919 & 1.0000 & 0.9967 & 1.0000 & 0.9973 \\
SR_EE & 1.0000 & 1.0000 & 1.0000 & 1.0000 & 1.0000 & 1.0000 & 1.0000 & 1.0000 & 1.0000 \\
SR_HE & 0.9657 & 0.9599 & 1.0000 & 1.0000 & 1.0000 & 1.0000 & 1.0000 & 1.0000 & 1.0000 \\
\hline
\end{tabular}

Note: CR - Czech Republic, HU - Hungary, PL - Poland, SR - Slovak Republic, EE - Environmental efficiency, HE - Healthcare efficiency.

Source: Own processing according to data collected from Eurostat (2020) and World Bank (2020). 
The most environmentally efficient country is Slovakia, which has been efficient for five years. In terms of health efficiency, it can be argued that none of the countries achieved significant inefficiencies, but the least efficient was Hungary. Similar results were recorded for the BCC models, see Table 4. The computed values are higher as the frontier efficiency is concave for the BCC model.

Table 5 shows the average values and selected characteristics of individual efficiencies for the entire V4 group.

Table 5

Statistical Characteristics of the Computed Efficiencies

\begin{tabular}{|l|c|c|c|c|c|c|}
\hline Variable & N & Mean & Std Dev & Median & Minimum & Maximum \\
\hline CCR_EE & 36 & 0.86316 & 0.12087 & 0.87428 & 0.62296 & 1.00000 \\
BCC_EE & 36 & 0.94978 & 0.05818 & 0.96450 & 0.77777 & 1.00000 \\
CCR_HE & 36 & 0.98548 & 0.01875 & 0.99598 & 0.93663 & 1.00000 \\
BCC_HE & 36 & 0.98918 & 0.01768 & 1.00000 & 0.93853 & 1.00000 \\
\hline
\end{tabular}

Source: Own processing according to data collected from Eurostat (2020) and World Bank (2020).

The worst efficiency results of the V4 countries were recorded in the case of the DEA CCR model of environmental efficiency. The best results have been achieved with the DEA BCC models of healthcare efficiency. Based on the above results, which indicate relatively good efficiency values, signs of a similar development of the individual calculated efficiencies of the V4 cluster can be observed. The following Table 6 shows the results of the correlation analysis performed using the Spearman correlation coefficient.

T a ble 6

Matrix of Spearman Correlation Coefficients

\begin{tabular}{|c|c|c|c|c|}
\hline \multicolumn{5}{|c|}{$\begin{array}{l}\text { Spearman Correlation Coefficients, } \mathrm{N}=36 \\
\text { Prob }>|\mathbf{r}| \text { under H0: Rho }=0\end{array}$} \\
\hline & CCR_EE & BCC_EE & CCR_HE & BCC_HE \\
\hline CCR_EE & 1.00000 & $\begin{array}{l}0.82308 \\
<.0001\end{array}$ & $\begin{array}{l}0.12748 \\
0.4587\end{array}$ & $\begin{array}{l}0.19955 \\
0.2433\end{array}$ \\
\hline BCC_HE & $\begin{array}{l}0.82308 \\
<.0001\end{array}$ & 1.00000 & $\begin{array}{l}0.08758 \\
0.6155\end{array}$ & $\begin{array}{l}0.03730 \\
0.8290\end{array}$ \\
\hline CCR_EE & $\begin{array}{l}0.12748 \\
0.4587\end{array}$ & $\begin{array}{l}0.08758 \\
0.6155\end{array}$ & 1.00000 & $\begin{array}{l}0.85469 \\
<.0001\end{array}$ \\
\hline BCC_HE & $\begin{array}{l}0.19955 \\
0.2433 \\
\end{array}$ & $\begin{array}{l}0.03730 \\
0.8290\end{array}$ & $\begin{array}{l}0.85469 \\
<.0001\end{array}$ & 1.00000 \\
\hline
\end{tabular}

Source: Own processing according to data collected from Eurostat (2020) and World Bank (2020).

Based on the results shown in Table 6, it can be stated that no relationship between environmental and health efficiency values has been demonstrated. In addition, the weak level of correlations was statistically insignificant. A statistically 
significant correlation was noted between the CCR and BCC models for environmental efficiency and between the CCR and BCC models for health efficiency. However, this fact is an expected result, which is a consequence of the mathematical construction of models. For completeness, we also present Table 7, which shows the results of Fisher's Z transformation. Based on the results, it can be observed that the corrected correlation coefficients are also insignificant and the null hypothesis that the correlation coefficient is equal to zero cannot be rejected in every comparison of environmental and health efficiency.

T a b l e 7

Statistical Characteristics of Computed Spearman Correlations

\begin{tabular}{|l|c|c|c|c|c|c|c|}
\hline Variable & $\begin{array}{c}\text { With } \\
\text { Variable }\end{array}$ & $\begin{array}{c}\text { Sample } \\
\text { Correlation }\end{array}$ & Fisher's z & $\begin{array}{c}\text { Correlation } \\
\text { Estimate }\end{array}$ & \multicolumn{2}{|c|}{ 95\% Confidence Limits } & $\begin{array}{c}\text { H0: Rho= Rhoo } \\
\text { p Value }\end{array}$ \\
\hline CCR_EE & BCC_EE & 0.82308 & 1.16629 & 0.81925 & 0.671429 & 0.904371 & $<0.0001$ \\
CCR_EE & CCR_HE & 0.12748 & 0.12818 & 0.12569 & -0.211851 & 0.436214 & 0.4615 \\
CCR_EE & BCC_HE & 0.19955 & 0.20226 & 0.19681 & -0.140832 & 0.493440 & 0.2453 \\
BCC_EE & CCR_HE & 0.08758 & 0.08780 & 0.08633 & -0.249272 & 0.403427 & 0.6140 \\
BCC_EE & BCC_HE & 0.03730 & 0.03732 & 0.03677 & -0.295337 & 0.360942 & 0.8303 \\
CCR_HE & BCC_HE & 0.85469 & 1.27332 & 0.85137 & 0.725860 & 0.922013 & $<0.0001$ \\
\hline
\end{tabular}

Source: Own processing according to data collected from Eurostat (2020) and World Bank (2020).

Subsequently, we proceeded to the second step of the two-step DEA analysis, in which it is necessary to calculate the resampled efficiencies. In this step, we have already considered only BCC efficiencies, which is justified in the previous section. The values of the resampled efficiencies are shown in the Table 8 .

Based on the above results, it can be stated that each DMU was less efficient during the given period than in the case of purely deterministic efficiency after considering the random error. For each DMU, we also calculated the confidence intervals in which the real efficiency will be located. These intervals are constructed with a $95 \%$ probability. We can observe lower value of efficiency in the case of Czech Republic in 2015, this was caused mostly because of higher value of Gross capital formation (as input) and lower value of GDP per capita (as output) in 2015. Since we have obtained stochastic efficiencies by this process, regression analysis can be performed using truncated regression. The results of models (3) and (4) are shown in Table 9.

Based on the results of the regression analysis, several interesting facts can be observed. The environmental efficiency model shows a higher predictive power. Both the R-squared values and the statistical significance of the individual variables are higher in the case of the environmental model. It can also be observed that Energy consumption and $\mathrm{PM}_{2.5}$ Air pollution have a negative effect on efficiency values. An interesting result is the positive effect of unemployment and the amount of waste produced. Recycling and organic farming have a positive 
but expected effect. Conversely, increasing sales of fertilizers, road and air transport is expected to lead to a reduction in environmental efficiency values. These statements also apply to the health efficiency model. The exceptions are the Unemployment rate and Energy consumption. These have the opposite effect as in the case of the environmental efficiency model. The problem, however, is the fact that most of the variables are not statistically significant for the model we created. If we look for common characteristics, it can be argued that variables related to transport and the organic farming ratio have a significant impact on both environmental and health efficiency.

T a ble 8

\section{Results of Double Bootstrap Procedures}

\begin{tabular}{|c|c|c|c|c|c|c|c|}
\hline \multirow[b]{2}{*}{ Year } & \multirow[b]{2}{*}{ Country } & \multicolumn{3}{|c|}{ Environmental BCC efficiency } & \multicolumn{3}{|c|}{ Healthcare BCC efficiency } \\
\hline & & $\begin{array}{c}\text { Double } \\
\text { bootstraped } \\
\text { efficiency }\end{array}$ & Lower CI & Upper CI & $\begin{array}{c}\text { Double } \\
\text { bootstraped } \\
\text { efficiency }\end{array}$ & Lower CI & Upper CI \\
\hline 2009 & $\mathrm{CR}$ & 0.9753 & 0.9529 & 1.0000 & 0.9966 & 0.9932 & 1.0000 \\
\hline 2010 & $\mathrm{CR}$ & 0.9702 & 0.9433 & 1.0000 & 0.9965 & 0.9932 & 1.0000 \\
\hline 2011 & $\mathrm{CR}$ & 0.9600 & 0.9244 & 1.0000 & 0.9906 & 0.9881 & 0.9944 \\
\hline 2012 & $\mathrm{CR}$ & 0.9345 & 0.9149 & 0.9618 & 0.9941 & 0.9917 & 0.9981 \\
\hline 2013 & $\mathrm{CR}$ & 0.9392 & 0.8872 & 1.0000 & 0.9895 & 0.9851 & 0.9975 \\
\hline 2014 & $\mathrm{CR}$ & 0.9001 & 0.8819 & 0.9236 & 0.9925 & 0.9854 & 1.0000 \\
\hline 2015 & $\mathrm{CR}$ & 0.7505 & 0.7313 & 0.7767 & 0.9891 & 0.9789 & 0.9968 \\
\hline 2016 & $\mathrm{CR}$ & 0.8604 & 0.8349 & 0.8882 & 0.9908 & 0.9826 & 1.0000 \\
\hline 2017 & $\mathrm{CR}$ & 0.9350 & 0.9160 & 0.9631 & 0.9966 & 0.9933 & 1.0000 \\
\hline 2009 & Hungary & 0.9450 & 0.9285 & 0.9655 & 0.9495 & 0.9371 & 0.9623 \\
\hline 2010 & Hungary & 0.9303 & 0.9151 & 0.9507 & 0.9113 & 0.9032 & 0.9197 \\
\hline 2011 & Hungary & 0.9492 & 0.9369 & 0.9675 & 0.9120 & 0.9056 & 0.9183 \\
\hline 2012 & Hungary & 0.9767 & 0.9574 & 0.9997 & 0.9463 & 0.9381 & 0.9543 \\
\hline 2013 & Hungary & 0.9184 & 0.9054 & 0.9378 & 0.9392 & 0.9344 & 0.9435 \\
\hline 2014 & Hungary & 0.8284 & 0.8157 & 0.8427 & 0.9632 & 0.9573 & 0.9676 \\
\hline 2015 & Hungary & 0.8320 & 0.8198 & 0.8483 & 0.9740 & 0.9674 & 0.9793 \\
\hline 2016 & Hungary & 0.8991 & 0.8867 & 0.9197 & 0.9484 & 0.9427 & 0.9530 \\
\hline 2017 & Hungary & 0.8508 & 0.8411 & 0.8668 & 0.9751 & 0.9709 & 0.9804 \\
\hline 2009 & Poland & 0.9046 & 0.8889 & 0.9364 & 0.9987 & 0.9974 & 1.0000 \\
\hline 2010 & Poland & 0.8698 & 0.8526 & 0.9015 & 0.9985 & 0.9971 & 1.0000 \\
\hline 2011 & Poland & 0.8316 & 0.8184 & 0.8535 & 0.9976 & 0.9952 & 1.0000 \\
\hline 2012 & Poland & 0.8844 & 0.8686 & 0.9141 & 0.9983 & 0.9967 & 1.0000 \\
\hline 2013 & Poland & 0.9097 & 0.8459 & 1.0000 & 0.9902 & 0.9884 & 0.9916 \\
\hline 2014 & Poland & 0.9180 & 0.8973 & 0.9461 & 0.9951 & 0.9903 & 1.0000 \\
\hline 2015 & Poland & 0.9093 & 0.8933 & 0.9440 & 0.9950 & 0.9933 & 0.9974 \\
\hline 2016 & Poland & 0.9363 & 0.9107 & 0.9835 & 0.9981 & 0.9965 & 1.0009 \\
\hline 2017 & Poland & 0.9291 & 0.8942 & 0.9837 & 0.9950 & 0.9931 & 1.0000 \\
\hline 2009 & SR & 0.9583 & 0.9234 & 1.0000 & 0.9389 & 0.9261 & 0.9467 \\
\hline 2010 & SR & 0.9618 & 0.9278 & 1.0000 & 0.9358 & 0.9217 & 0.9446 \\
\hline 2011 & SR & 0.9608 & 0.9252 & 1.0000 & 0.9785 & 0.9604 & 0.9898 \\
\hline 2012 & SR & 0.9775 & 0.9572 & 1.0000 & 0.9772 & 0.9654 & 0.9850 \\
\hline 2013 & SR & 0.9654 & 0.9349 & 1.0000 & 0.9777 & 0.9703 & 0.9848 \\
\hline 2014 & SR & 0.9642 & 0.9321 & 1.0000 & 0.9830 & 0.9752 & 0.9904 \\
\hline 2015 & SR & 0.9934 & 0.9874 & 1.0000 & 0.9824 & 0.9705 & 0.9930 \\
\hline 2016 & SR & 0.9931 & 0.9867 & 1.0000 & 0.9792 & 0.9630 & 0.9933 \\
\hline 2017 & SR & 0.9903 & 0.9817 & 1.0000 & 0.9884 & 0.9783 & 1.0000 \\
\hline
\end{tabular}

Source: Own processing according to data collected from Eurostat (2020) and World Bank (2020). 
Based on the above results, it can be confirmed that especially in the case of the environmental efficiency model, other than traditional indicators of DEA models can be tested to compare the examined countries, such as organic farming, production of "bio" products, circular economy indicators, production of renewable energy and others.

T a b l e 9

\section{Results of Truncated Regression Models}

\begin{tabular}{|c|c|c|c|c|}
\hline \multirow{2}{*}{$\begin{array}{l}\text { Variable } \\
\text { Intercept }\end{array}$} & \multicolumn{2}{|c|}{$\begin{array}{c}\text { Double bootstrapped DEA BCC } \\
\text { environmental efficiency (3) }\end{array}$} & \multicolumn{2}{|c|}{$\begin{array}{c}\text { Double bootstrapped DEA } \\
\text { BCC healthcare efficiency (4) }\end{array}$} \\
\hline & 1.10675000 & $* * *$ & 1.16340000 & $* * *$ \\
\hline Energy consumption & -0.00557150 & & 0.00906380 & \\
\hline $\mathrm{PM}_{2.5}$ air pollution & -0.02907900 & $* * *$ & -0.00555970 & \\
\hline Unemployment & 0.02507100 & $* * *$ & -0.00573920 & \\
\hline Waste generated & 0.00111030 & $* * *$ & 0.00050009 & \\
\hline Recycling - composting and digestion & 0.00752940 & $* * *$ & 0.00251340 & \\
\hline Area - organic farming & 0.02685800 & $* * *$ & 0.01257000 & $* *$ \\
\hline Sales of fertilisers & -0.00000003 & & -0.00000002 & \\
\hline Freight transport & -0.00959490 & $* * *$ & -0.00385190 & $* *$ \\
\hline Air transport & -0.01730100 & $* * *$ & -0.01200000 & $*$ \\
\hline sigma & 0.03120500 & $* * *$ & 0.01478000 & $* * *$ \\
\hline R-squared & 0.7117 & & 0.6063 & \\
\hline Log-likelihood & 90.0300 & & 138.8500 & \\
\hline
\end{tabular}

Source: Own processing according to data collected from Eurostat (2020) and World Bank (2020).

\section{Discussion}

The aim of this study was to examine the possible relationship between environmental and health care efficiency in the V4 countries and to reveal the common determinants of these efficiencies. Based on the results of the analysis using DEA models, it can be stated that no such relationship for the observed period, using the given variables was demonstrated. More importantly, we were able to propose regression models that appear to be suitable for describing the determinants of environmental efficiency and, to some extent, for describing health care efficiency. Since this article is unique both by the object of research and by comparing the two types of efficiency, it is quite difficult to evaluate it with respect to the existing literature.

The reason for the absence of a significant relationship between environmental and health efficiency may be the short time period of the analysis performed. However, this period was selected due to the unavailability of data. Some variables seemed to be very suitable as inputs and outputs of DEA models, their inconsistency did not allow their use. Another reason may be the relatively significantly better efficiency of healthcare. However, this fact does not mean that the health care of the V4 countries is at an excellent level. This means that they are 
on a similar level. On the contrary, in the case of environmental efficiency, we find greater differences between countries. The V4 countries are still more focused on industry than on services or the knowledge economy, in some countries this implies higher production of emissions. Also, some countries are implementing resources to improve the environment more slowly or less efficient. Increasing environmental efficiency brings increased costs for both the economy and businesses, thus reducing their ability to compete with other countries where strict emission limits do not apply.

The positive fact is that some determinants are common to both environmental and health efficiency. This means that focusing on their improvement will bring positive results for both the environment and health care, and ultimately for the health of the population. These determinants include indicators related to transport. As there is no presumption of reducing the volume of both road, air and other transport, it is necessary to make it more efficient. In such a way as to pollute the environment as little as possible. These processes take place at the level of EU countries and thus also at the level of V4 countries.

The trend that organic farms can be useful not only for the health of the population but also for improving the overall state of the environment has also been confirmed. The trend of organic products is widespread, even in the V4 countries. Although perhaps to a lesser extent than in countries such as France, Germany or other more developed EU countries. However, its importance is growing, and not only financial but also human resources should be directed to this area.

\section{Conclusion}

As the analysis and discussion performed have shown the common features of countries in the fields of environment and health, it is possible to implement the results of this study into the policies of these countries. Although the EU should act as a whole, the local characteristics of the selected regions cannot be forgotten. Because these have been proven by efficiency assessment models, which did not differ much between the countries identified, both in terms of environmental efficiency and even more so in terms of health efficiency. Research examining larger political groupings shows significant differences in efficiency values. The search for such groupings can contribute to the understanding of the development of the given countries and specialize assistance to these regions. This must also be considered when directing resources from the European Structural Funds.

The countries researched would also certainly help each other to improve the areas assessed, through joint negotiations between the V4 countries. Mutual assistance and advice will also help other countries, given the similarity of development 
processes. It is therefore clear that this cooperation should also be developed in the areas of health and the environment.

The health care systems of these countries are very similar. This is reflected in the common history of these countries, but it can be said that their health care is slightly underfunded regarding the more developed economies of the EU and the world. The continuous and consistent development of science and research, which will not be affected by the change of government, but will be in the interest of society as a whole, which the state will protect and significantly not only financially support, may help the joint faster catching up of more developed countries. It must also be emphasized that funding for science and research is not as high in these countries as in knowledge-based economies.

We have proved, that there is no significant correlation between health care and environmental efficiency among selected countries. On the other hand, we have found determinants, which can be used to explain both types of efficiency.

If this research was carried out in a larger political grouping, such as the EU, the OECD and others, it is possible that a common trend towards environmental and health efficiency would be revealed. It is important to realize that the sample on which we conducted the research is not large, as it is only 4 countries. Research is more specialized in either individual countries or larger entities. It is the assessment of the development of environmental and health efficiency of individual countries but also large groups that is an opportunity for further research in this area, which can bring interesting results.

\section{References}

AFONSO, A. - ST. AUBYN, M. (2011): Assessing Health Efficiency across Countries with a Two-Step and Bootstrap Analysis. Applied Economics Letters, 18, No. 15, pp. 1427 - 1430. Available at: 〈https://doi.org/10.1080/13504851.2010.541149>.

ALSALEH, M. - ABDUL-RAHIM, A. S. - MOHD-SHAHWAHID, H. O. (2017): Determinants of Technical Efficiency in the Bioenergy Industry in the EU28 Region. Renewable and Sustainable Energy Reviews, 78, pp. 1331 - 1349. Available at: <https://doi.org/10.1016/j.rser.2017.04.049>.

ARBOLINO, R. - CARLUCCI, F. - DE SIMONE, L. - IOPPOLO, G. - YIGITCANLAR, T. (2018): The Policy Diffusion of Environmental Performance in the European Countries. Ecological Indicators, 89, pp. 130 - 138. Available at: 〈https://doi.org/10.1016/j.ecolind.2018.01.062〉.

BIAN, Y. - YANG, F. (2010): Resource and Environment Efficiency Analysis of Provinces in China: A DEA Approach Based on Shannon's Entropy. Energy Policy, 38, No. 4, pp. 1909 1917. Available at: 〈https://doi.org/10.1016/j.enpol.2009.11.071〉.

CAI, W. - LI, G. (2018): The Drivers of Eco-Innovation and Its Impact on Performance: Evidence From China. Journal of Cleaner Production, 176, pp. 110 - 118. Available at: $\langle$ https://doi.org/10.1016/j.jclepro.2017.12.109>.

CHARNES, A. - CLARK, C. T. - COOPER, W. W. - GOLANY, B. (1984): A Developmental Study of Data Envelopment Analysis in Measuring the Efficiency of Maintenance Units in the U.S. Air Forces. Annals of Operations Research, 2, No. 1, pp. 95 - 112. Available at: <https://doi.org/10.1007/BF01874734>. 
CHARNES, A. - COOPER, W. W. - LEWIN, A. Y. - SEIFORD, L. M. (2013): Data Envelopment Analysis: Theory, Methodology, and Applications. Springer Science and Business Media.

CHUANG, C.-L. - CHANG, P.-C. - LIN, R.-H. (2011): An Efficiency Data Envelopment Analysis Model Reinforced by Classification and Regression Tree for Hospital Performance Evaluation. Journal of Medical Systems, 35, No. 5, pp. 1075 - 1083. Available at: <https://doi.org/10.1007/s10916-010-9598-5>.

COOPER, W. W. - SEIFORD, L. M. - TONE, K. (2007): Data Envelopment Analysis: A Comprehensive Text with Models, Applications, References and DEA-Solver Software. 2nd ed. New York: Springer US. Available at: //www.springer.com/la/book/9780387452814>.

DUMAN, Y. S. - KASMAN, A. (2018): Environmental Technical Efficiency in EU Member and Candidate Countries: A Parametric Hyperbolic Distance Function Approach. Energy, 147, pp. 297 - 307. Available at: 〈https://doi.org/10.1016/j.energy.2018.01.037>.

EMROUZNEJAD, A. - YANG, G. (2018): A Survey and Analysis of the First 40 Years of Scholarly Literature in DEA: 1978 - 2016. Socio-Economic Planning Sciences, 61, pp. 4 - 8. Available at: 〈https://doi.org/10.1016/j.seps.2017.01.008>.

EUROSTAT (2020): Database - Eurostat. Available at: $<$ https://ec.europa.eu/eurostat/data/database>.

FARRELL, M. J. (1957): The Measurement of Productive Efficiency. Journal of the Royal Statistical Society. Series A (General), 120, No. 3, pp. 253. Available at: <https://doi.org/10.2307/2343100>.

GRAUSOVÁ, M. - HUŽVÁR, M. - ŠTRANGFELDOVÁ, J. (2014): Healthcare Systems Efficiency in the Visegrád Group. International Scientific Conference. 17-th AMSE. Applications of Mathematics in Economics. International Scientific Conference: Poland, pp. 27 - 31 August, 2014. [Conference Proceedings Full Text Papers.] Available at: <https://doi.org/10.15611/amse.2014.17.11>.

HADAD, S. - HADAD, Y. - SIMON-TUVAL, T. (2013): Determinants of Healthcare System's Efficiency in OECD Countries. The European Journal of Health Economics, 14, No. 2, pp. 253 - 265. Available at: 〈https://doi.org/10.1007/s10198-011-0366-3>.

HALKOS, G. E. - TZEREMES, N. G. (2009): Exploring the Existence of Kuznets Curve in Countries' Environmental Efficiency Using DEA Window Analysis. Ecological Economics, 68, No. 7, pp. 2168 - 2176. Available at: 〈https://doi.org/10.1016/j.ecolecon.2009.02.018>.

HALKOS, G. E. - TZEREMES, N. G. (2011): A Conditional Nonparametric Analysis for Measuring the Efficiency of Regional Public Healthcare Delivery: An Application to Greek Prefectures. Health Policy, 103, No. 1, pp. 73 - 82. Available at: <https://doi.org/10.1016/j.healthpol.2010.10.021>

HALKOS, G. - PETROU, K. N. (2019): Analysing the Energy Efficiency of EU Member States: The Potential of Energy Recovery from Waste in the Circular Economy. Energies, 12, No. 19, pp. 3718. Available at: 〈https://doi.org/10.3390/en12193718>.

HOLLINGSWORTH, B. (2003): Non-Parametric and Parametric Applications Measuring Efficiency in Health Care. Health Care Management Science, 6, pp. 203 - 218.

HSU, Y.-C. (2013): The Efficiency of Government Spending on Health: Evidence from Europe and Central Asia. The Social Science Journal, 50, No. 4, pp. 665 - 673. Available at: <https://doi.org/10.1016/j.soscij.2013.09.005>.

JI, X. - YAO, Y. - LONG, X. (2018): What Causes PM2.5 Pollution? Cross-Economy Empirical Analysis from Socioeconomic Perspective. Energy Policy, 119, pp. 458 - 472. Available at: $<$ https://doi.org/10.1016/j.enpol.2018.04.040>.

KAYA SAMUT, P. - CAFRI, R. (2016): Analysis of the Efficiency Determinants of Health Systems in OECD Countries by DEA and Panel Tobit. Social Indicators Research, 129, No. 1, pp. 113 - 132. Available at: 〈https://doi.org/10.1007/s11205-015-1094-3>.

KOIŠOVÁ, E. - GRMANOVÁ, E. - ŠKROVÁNKOVÁ, K. - KOSTROVÁ, J. (2019): Competitiveness of Regions in the Visegrad Group Countries. Engineering Economics, 30, No. 2, pp. 203 - 210. Available at: 〈https://doi.org/10.5755/j01.ee.30.2.21746>. 
KOUNETAS, K. (2015): Heterogeneous Technologies, Strategic Groups and Environmental Efficiency Technology Gaps for European Countries. Energy Policy, 83, pp. 277 - 287. Available at: <https://doi.org/10.1016/j.enpol.2015.01.036>.

LACKO, R. - HAJDUOVÁ, Z. (2018): Determinants of Environmental Efficiency of the EU Countries Using Two-Step DEA Approach. Sustainability, 10, No. 10, pp. 3525. Available at: <https://doi.org/10.3390/su10103525>.

LI, M. - WANG, Q. (2014): International Environmental Efficiency Differences and Their Determinants. Energy, 78, pp. $411-420$. Available at: $<$ https://doi.org/10.1016/j.energy.2014.10.026>.

LIU, J. S. - LU, L. Y. Y. - LU, W.-M. - LIN, B. J. Y. (2013): A Survey of DEA Applications. Omega, 41, No. 5, pp. 893 - 902. Available at: 〈https://doi.org/10.1016/j.omega.2012.11.004〉.

LIU, L. - CHEN, Y. - WU, T. - LI, H. (2018): The Drivers of Air Pollution in the Development of Western China: The Case of Sichuan Province. Journal of Cleaner Production, 197, pp. 1169 1176. Available at: <https://doi.org/10.1016/j.jclepro.2018.06.260>.

MADALENO, M. - MOUTINHO, V. - ROBAINA, M. (2016): Economic and Environmental Assessment: EU Cross-country Efficiency Ranking Analysis. Energy Procedia, 106, pp. 134 - 154. Available at: 〈https://doi.org/10.1016/j.egypro.2016.12.111〉.

MARDANI, A. - ZAVADSKAS, E. K. - STREIMIKIENE, D. - JUSOH, A. - KHOSHNOUDI, M. (2017): A Comprehensive Review of Data Envelopment Analysis (DEA) Approach in Energy Efficiency. Renewable and Sustainable Energy Reviews, 70, pp. 1298 - 1322. Available at: <https://doi.org/10.1016/j.rser.2016.12.030>.

McDONALD, J. (2009): Using Least Squares and Tobit in Second Stage DEA Efficiency Analyses. European Journal of Operational Research, 197, No. 2, pp. 792 - 798. Available at: <https://doi.org/10.1016/j.ejor.2008.07.039>.

MOUTINHO, V. - MADALENO, M. - ROBAINA, M. (2017): The Economic and Environmental Efficiency Assessment in EU Cross-Country: Evidence from DEA and Quantile Regression Approach. Ecological Indicators, 78, pp. 85 - 97. Available at: <https://doi.org/10.1016/j.ecolind.2017.02.042>.

NAYAR, P. - OZCAN, Y. A. (2008): Data Envelopment Analysis Comparison of Hospital Efficiency and Quality. Journal of Medical Systems, 32, No. 3, pp. 193 - 199. Available at: <https://doi.org/10.1007/s10916-007-9122-8>.

O'NEILL, L. - RAUNER, M. - HEIDENBERGER, K. - KRAUS, M. (2008): A Cross-National Comparison and Taxonomy of DEA-Based Hospital Efficiency Studies. Socio-Economic Planning Sciences, 42, No. 3, pp. 158 - 189. Available at: 〈https://doi.org/10.1016/j.seps.2007.03.001〉.

SHI, G.-M. - BI, J. - WANG, J.-N. (2010): Chinese Regional Industrial Energy Efficiency Evaluation Based on a DEA Model of Fixing Non-Energy Inputs. Energy Policy, 38, No. 10, pp. 6172 - 6179. Available at: 〈https://doi.org/10.1016/j.enpol.2010.06.003〉.

SIMAR, L. - WILSON, P. W. (2007): Estimation and Inference in Two-Stage, Semi-Parametric Models of Production Processes. Journal of Econometrics, 136, No. 1, pp. 31 - 64. Available at: <https://doi.org/10.1016/j.jeconom.2005.07.009>.

SUEYOSHI, T. - GOTO, M. (2010): Should the US Clean Air Act Include CO2 Emission Control? Examination By Data Envelopment Analysis. Energy Policy, 38, No. 10, pp. $5902-5911$. Available at: 〈https://doi.org/10.1016/j.enpol.2010.05.044>.

TAJUDEEN, I. A. - WOSSINK, A. - BANERJEE, P. (2018): How Significant is Energy Efficiency to Mitigate CO2 Emissions? Evidence from OECD Countries. Energy Economics, 72, pp. 200 - 221. Available at: 〈https://doi.org/10.1016/j.eneco.2018.04.010>.

THE WORLD BANK (2020): World Bank Open Data. Washington, DC: The World Bank. Available at: <https://data.worldbank.org/>

WANG, K. - YU, S. - ZHANG, W. (2013): China's Regional Energy and Environmental Efficiency: A DEA Window Analysis Based Dynamic Evaluation. Mathematical and Computer Modelling, 58, No. 5, pp. 1117 - 1127. Available at: <https://doi.org/10.1016/j.mcm.2011.11.067>. 
WANG, X. - HAN, L. - YIN, L. (2016): Environmental Efficiency and Its Determinants for Manufacturing in China. Sustainability, 9, No. 1, pp. 47. Available at: $<$ https://doi.org/10.3390/su9010047>.

WOO, C. - CHUNG, Y. - CHUN, D. - SEO, H. - HONG, S. (2015): The Static and Dynamic Environmental Efficiency of Renewable Energy: A Malmquist Index Analysis of OECD Countries. Renewable and Sustainable Energy Reviews, 47, pp. 367 - 376. Available at: <https://doi.org/10.1016/j.rser.2015.03.070>.

YANG, L. - OUYANG, H. - FANG, K. - YE, L. - ZHANG, J. (2015): Evaluation of Regional Environmental Efficiencies in China Based on Super-Efficiency-DEA. Ecological Indicators, 51, pp. 13 - 19. Available at: 〈https://doi.org/10.1016/j.ecolind.2014.08.040>.

ZHOU, P. - ANG, B. W. - POH, K. L. (2008): Measuring Environmental Performance under Different Environmental DEA Technologies. Energy Economics, 30, No. 1, pp. 1 - 14. Available at: 〈https://doi.org/10.1016/j.eneco.2006.05.001〉. 